\title{
Élargissement du choix des contraceptifs
}

\author{
Heather Clark \\ Population Council \\ Saumya RamaRao \\ Population Council \\ Catherine Unthank \\ Population Council \\ Kazuyo Machiyama \\ Nandita Thatte
}

Follow this and additional works at: https://knowledgecommons.popcouncil.org/departments_sbsr-rh

Part of the Social and Behavioral Sciences Commons

How does access to this work benefit you? Let us know!

\section{Recommended Citation}

Clark, Heather, Saumya Ramarao, Catherine Unthank, Kazuyo Machiyama, and Nandita Thatte. 2018.

"Élargissement du choix des contraceptifs," Résumés des preuves sur la planification familiale. New York: Population Council. 


\section{Élargissement du choix des contraceptifs}

\section{$\rightarrow$ « L'élargissement des options et des choix} pour les femmes les plus pauvres et les adolescentes est la chose la plus importante que nous faisons. En les habilitant à prendre leurs propres décisions concernant le moment et l'espacement des grossesses, nous ouvrons une voie importante vers leur sécurité économique et leur indépendance, ainsi qu'à la réalisation de tous les objectifs du développement durable. »

-Dr. Natalia Kanem, Directrice exécutive du Fonds des Nations unies pour la population

Environ 214 millions de femmes en âge de procréer dans les régions en développement qui veulent éviter la grossesse n'utilisent pas de méthode contraceptive moderne et sont considérées comme ayant un besoin non satisfait (1). L'élargissement des options contraceptives pour la planification familiale volontaire est essentiel pour répondre à ce besoin pour plusieurs raisons. Premièrement, les besoins peuvent varier tout au long du cycle de la vie reproductive, et les individus ont des désirs différents selon leur situation personnelle et familiale ; 25 $\%$ des femmes souhaitent espacer leurs grossesses, ce qui reflète le besoin de méthodes à court terme ou réversibles, contre à $14 \%$ des

L'utilisation de la contraception moderne augmente lorsque davantage de méthodes sont disponibles : analyse des preuves de 1982-2009

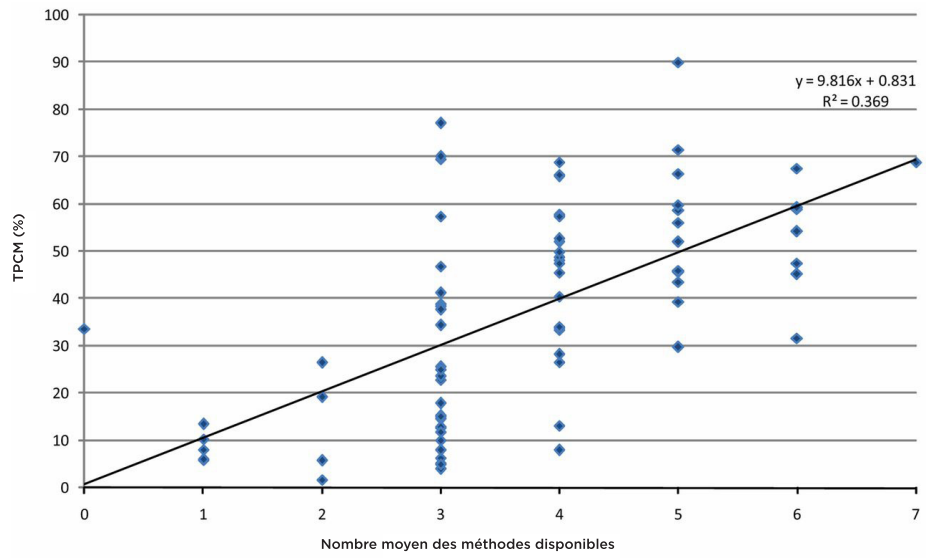

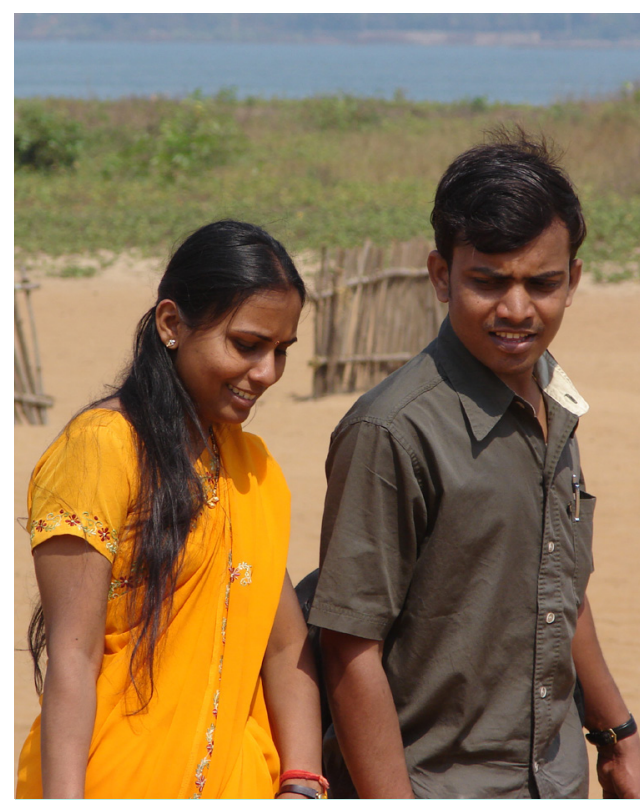

CONSIDÉRATIONS POLITIQUES ET PROGRAMMATIQUES

Établir et maintenir le bon fonctionnement des chaînes d'approvisionnement pour un choix élargi de contraceptifs:

\section{$\rightarrow$ Introduire de nouvelles} technologies contraceptives pour satisfaire les diverses préférences des femmes et de leurs partenaires.

$\rightarrow$ Surmonter les obstacles d'accès aux méthodes existantes telles que les circonstances économiques et sociales défavorables, y compris les normes de genre.

$\rightarrow$ Maximiser le potentiel de choix élargis en matière de contraception en assurant une chaîne d'approvisionnement solide et en améliorant la formation et le conseil des prestataires. 
femmes qui souhaitent cesser de procréer et limiter ainsi les grossesses futures (2). Deuxièmement, certaines femmes cessent de recourir à la contraception même si elles veulent éviter la grossesse $(3,4)$. Les raisons de l'interruption comprennent les effets secondaires, les mythes, l'échec de la contraception ou l'environnement de service, y compris la qualité du service et la disponibilité d'un choix suffisant de méthodes (4).

Un large choix de contraceptifs répondra aux besoins de certaines femmes qui ont interrompu leur utilisation si elles ont la possibilité de passer à une autre méthode. La disponibilité élargie de méthodes peut contribuer à réduire de $8 \%$ l'interruption de la contraception (5). Troisièmement, les trois cinquièmes des femmes (62 \%) dont les besoins ne sont pas satisfaits n'ont jamais utilisé de contraceptifs (5), et une gamme plus large d'options peut être suffisamment attrayante pour que certaines femmes tentent la contraception. L'ajout d'une nouvelle méthode à la combinaison des méthodes disponibles peut augmenter la prévalence contraceptive de huit points de pourcentage (6). Enfin, l'élargissement du choix des méthodes peut assurer que les méthodes sont disponibles pour les personnes qui veulent se protéger contre la grossesse et les infections sexuellement transmissibles, y compris le VIH.

\section{CONSIDÉRATIONS RELATIVES À L'EXPANSION DU CHOIX DE CONTRACEPTIFS}

\section{$\rightarrow$ STRATÉGIES D’EXPANSION DES OPTIONS CONTRACEPTIVES}

Des stratégies éprouvées pour élargir les options contraceptives comprennent l'introduction de nouveaux contraceptifs dans les programmes de planification familiale, l'élargissement de l'accès aux méthodes existantes et l'élargissement de la base de fournisseurs.

\section{Introduire de nouvelles technologies contraceptives}

De nouvelles technologies contraceptives ont été mises au point pour satisfaire les différentes préférences des femmes et de leurs partenaires, et pour surmonter les difficultés que représente la fourniture de certaines méthodes dans des contextes à faibles ressources. Par exemple, des études ont montré que le DMPA SC en Uniject (Sayana ${ }^{\circledR}$ Press) peut être fourni par des cadres de santé communautaires et par des utilisateurs eux-mêmes, réduisant ainsi la dépendance à l'égard des systèmes de santé $(7,8)$. Des études suggèrent également que les femmes trouvent que Sayana ${ }^{\oplus}$ Press est un choix contraceptif réalisable et acceptable qui est en cours d'introduction, de mise à l'échelle ou piloté dans plus de 15 pays PF2O2O $(9,10,11,12)$. Parmi les autres catégories de produits, il y a les anneaux vaginaux que les femmes peuvent insérer elles-mêmes et qui peuvent être proposés dans différents points d'approvisionnement allant de pharmacies à des établissements de niveau tertiaire $(13,14)$. Le système intra-utérin à libération de lévonorgestrel (LNG) et les nouvelles technologies telles qu'un implant biodégradable, des injectables à longue durée d'action, des micro-puces contraceptives télécommandées, et des technologies de prévention polyvalentes (TPP) pour la contraception, le VIH et/ou la prévention des IST promettent un choix encore plus large de méthodes. En plus de la vasectomie et de la ligature des trompes, des options contraceptives permanentes plus abordables, plus sûres et plus simples pour les femmes et les hommes devraient également être envisagées, étant donné qu'il existe un besoin important non satisfait de limiter les grossesses.

\section{Élargir l'accès aux méthodes existantes}

Le fait de surmonter les obstacles aux méthodes existantes telles que les politiques, la géographie et les conditions économiques et sociales défavorables, y compris les normes restrictives en matière de genre, élargira l'accès aux méthodes existantes. Par exemple, l'élargissement de l'accès aux implants par le biais de programmes de distribution mobile et la réduction du prix grâce à une garantie en volume ont entraîné des augmentations significatives de l'utilisation $(15,16)$. De même, les obstacles socioculturels à la prestation de services aux adolescents peuvent être surmontés de plusieurs manières, notamment par l'éducation sexuelle complète, la programmation soucieuse de l'égalité entre les sexes, les services adaptés aux adolescents et la fourniture de contraceptifs par l'intermédiaire de différents points de service (17).

\section{Élargir la base des fournisseurs}

Le transfert de tâches ou le partage des tâches est la redistribution du counseling et de la fourniture de services sur la contraception entre les différentes catégories d'agents de santé. Par exemple, permettre aux agents de santé communautaire (ASC) de fournir des contraceptifs injectables, comme Sayana ${ }^{\circledR}$ Press et des implants, a élargi l'accès aux deux méthodes et leur utilisation volontaire dans de nombreux pays $(18,19,20)$. L'extension de la fourniture au personnel des pharmacies a également accru l'accès aux pilules contraceptives orales, y compris celles pour la contraception d'urgence et les contraceptifs injectables, en particulier pour les populations mal desservies (21). L'augmentation du nombre de fournisseurs permet aux utilisateurs de choisir des points de prestation de services pratiques et accessibles. Enfin, les contraceptifs initiés par l'utilisateur, comme les méthodes de sensibilisation à la fertilité et les pilules, permettent de plus en plus aux femmes d'utiliser de façon indépendante les contraceptifs, ce qui en accroît l'accès.

\section{$\rightarrow$ MAXIMISER LE POTENTIEL D'EXPANSION DES OPTIONS CONTRACEPTIVES}

Les actions suivantes sont essentielles pour garantir le succès de ces stratégies et conduire à un meilleur accès, à l'utilisation et au maintien de la contraception:

\section{Assurer une chaîne d'approvisionnement solide}

Il est essentiel d'assurer la disponibilité des produits contraceptifs aux points de soins proches des clients. Des chaînes d'approvisionnement solides qui éliminent les ruptures de stocks et impliquent plusieurs fabricants pour assurer la qualité des produits contraceptifs à faible coût sont essentiels à l'adoption et à la continuation (22).

\section{Améliorer la formation et le conseil des prestataires}

Les fournisseurs doivent être formés à la prestation de services axés sur la clientèle pour une gamme de méthodes tant dans les milieux cliniques que dans les communautés $(23,24)$. Un counseling complet et précis permet aux clients d'en apprendre davantage sur les options 


\begin{tabular}{|c|c|c|c|c|c|c|}
\hline \multirow[t]{2}{*}{ Méthode } & \multicolumn{2}{|c|}{ Sexe } & \multicolumn{2}{|c|}{ Hormonale } & \multicolumn{2}{|c|}{ À l’initiative du prestataire } \\
\hline & Femme & Homme & Oui & Non & Oui & Non \\
\hline \multicolumn{7}{|c|}{ Contraceptifs à courte durée d'action } \\
\hline Préservatif masculin & & $x$ & & $x$ & & $x$ \\
\hline Préservatif féminin & $x$ & & & $X$ & & $X$ \\
\hline $\begin{array}{l}\text { Injectables (DMPA-- } \\
\text { SC en Uniject) }\end{array}$ & $x$ & & $x$ & & & $x$ \\
\hline Injectables (autres) & $x$ & & $x$ & & $x$ & \\
\hline Pilules & $x$ & & $x$ & & & $x$ \\
\hline Anneaux vaginaux & $x$ & & $x$ & & & $x$ \\
\hline & $x$ & & & $x$ & & $x$ \\
\hline \multicolumn{7}{|c|}{ Méthodes à longue durée d'action réversibles (MLDAR) } \\
\hline Implants & $x$ & & $x$ & & $x$ & \\
\hline DIU - hormonal & $x$ & & $x$ & & $x$ & \\
\hline DIU - cuivre & $x$ & & & $x$ & $x$ & \\
\hline \multicolumn{7}{|c|}{ Contraception permanente } \\
\hline Ligature des trompes & $x$ & & & $X$ & $x$ & \\
\hline Vasectomie & & $x$ & & $x$ & $x$ & \\
\hline
\end{tabular}

contraceptives, y compris la gestion des effets secondaires, des avantages et la possibilité de changer de méthode (25). Les utilisateurs peuvent alors choisir un produit contraceptif qui convient le mieux à leurs besoins, ou changer les méthodes s'ils le souhaitent. L'absence de connaissances des fournisseurs sur les méthodes sous-utilisées, comme la vasectomie sans scalpel, pourrait également accroître l'accès aux contraceptifs (26).

\section{Générer la demande}

Même avec une base de fournisseurs bien informée, une combinaison élargie de méthodes ne peut atteindre son plein potentiel qu'avec la demande continue des utilisateurs. Par exemple, malgré les avantages bien reconnus de la vasectomie, y compris son efficacité élevée, sa commodité, sa permanence, la facilité relative de la prestation, le peu d'effets secondaires et les niveaux élevés de satisfaction, l'utilisation de la méthode est marquée par une faible demande chez les hommes (27). L'expérience du programme de planification familiale suggère que la production de la demande par le biais de campagnes médiatiques et de services mobiles peut accroître l'utilisation des contraceptifs et l'intention d'utiliser des contraceptifs modernes en améliorant les connaissances et les attitudes des communautés et des individus et en encourageant la communication des partenaires (28). Les utilisateurs de contraceptifs satisfaits qui parlent aux autres de leur expérience sont également très efficaces pour générer la demande et changer les normes sociales liées à la planification familiale volontaire (28).

\section{ATTEINDRE LES OBJECTIFS DU PROGRAMME PF2020 ET AU-DELÀ}

Le fait d'élargir l'éventail des options contraceptives aide les individus à faire des choix adaptés à leurs besoins et à leur situation, leur permet de passer d'une méthode à l'autre s'ils le souhaitent, et reflète un programme axé sur la qualité et les droits.

\section{RÉFÉRENCES}

$1 \quad$ Guttmacher Institute. 2017. "Adding It Up: Investing in Contraception and Maternal and Newborn Health, 2017." New York: Guttmacher Institute. https://www.guttmacher. org/fact-sheet/ adding-it-up-contraception-mnh-2017.

2 Van Lith, L.M., M. Yahner, et L. Bakamjian. "Women's growing desire to limit births in sub-Saharan Africa: meeting the challenge," Global Health, Science and Practice. (1): 97-107. doi:10.9745/ GHSP-D-12-00036.

3 Ali, M.M., J.G. Cleland, et I.H. Shah. 2012. Causes and Consequences of Contraceptive Discontinuation: Evidence from 60 Demographic and Health Surveys. OMS: Genève, Suisse.

4 FP2O2O and Population Council. 2015. "Contraceptive discontinuation: Reasons, challenges and solutions.

5 Jain, A., F. Obare, S. RamaRao, et I. Askew. 2013. "Reducing unmet need by supporting women with met need," International Perspectives on Sexual and Reproductive Health, 39(3): 133-141. doi: 10.1363/3913313.

6 Ross, J. et J. Stover. 2013. "Use of modern contraception increases when more methods become available: Analysis of evidence from 1982-2009," Global Health Science and Practice, 1(2): 203-212.

7 Burke, H.M, M.P. Mueller, C. Packer, B. Perry, L. Bufumbo, D. Mbengue, B.M. Daff, et A. Mbonye. 2014. "Provider acceptability of Sayana ${ }^{\circledR}$ Press: Results from community health workers and clinic based providers in Uganda and Senegal," Contraception, 89(5): 368-373.

8 Burke, H.M., M.P. Mueller, B. Perry, C. Packer, L. Bufumbo, C. Mbengue, I. Mall, et B. Mamadou. 2014. "Observational study of the 
acceptability of Sayana ${ }^{\circledast}$ Press among intramuscular DMPA users in Uganda and Senegal," Contraception, 89(5): 385-395.

9 Cover. J., A. Namagembe, J. Tumusiime, D. Nsangi, J. Lim, et D. Nakiganda-Busiku. 2018. "Continuation of injectable contraception when self-injected vs. administered by a facility-based health worker: A nonrandomized, prospective cohort study in Uganda" Contraception, https://doi.org/10.1016/j.contraception.2018.03.032

10 Cover, J., M. Ba, J. Lim, J.K. Drake, et B.M. Daff. 2019. "Evaluating the feasibility and acceptability of self-injection of subcutaneous depot medroxyprogesterone acetate (DMPA) in Senegal: A prospective cohort study," Contraception, 96(3): 203-210.

11 Shelton, J.D. et V. Halpern. 2014. "Subcutaneous DMPA: A better lower dose approach," Contraception, 89(5): 341-343.

12 "FP2O2O in Countries: Delivering High-Quality and Rights-Based Services." http://progress.familyplanning2020.org/en/fp2020-incountries/delivering-high-quality-and-rights-based-services

13 Merkatz, R.B, M. Plagianos, E. Hoskin, M. Cooney, P.C. Hewett, et B.S. Mensch. 2014. "Acceptability of the nestorone ${ }^{\circledR} /$ ethinyl estradiol contraceptive vaginal ring: Development of a model; implications for introduction," Contraception, 90(5): 514-521.

14 RamaRao, S., H. Clark, R. Merkatz, H. Sussman, et R. Sitruk-Ware. 2013. "Progesterone vaginal ring: Introducing a contraceptive to meet the needs of breastfeeding women," Contraception, 88(5): 591-598.

15 Duvall, S., S. Thurston, M. Weinberger, O. Nuccio, et Nomi FuchsMontgomery. 2014. "Scaling up delivery of contraceptive implants in sub-Saharan Africa," Global Health Science and Practice, 2(1): 72-92.

16 Christofield, M. et M. Lacoste. 2016. "Accessible contraceptive implant removal services: An essential element of quality service delivery and scale-up," Global Health Science and Practice, 4(3): 366-372.

17 Chandra-Mouli, V., D.R. McCarraher, S.J. Phillips, N.E. Williamson, et G. Hainsworth. 2014 "Contraception for adolescents in low and middle income countries: needs, barriers, and access," Reproductive Health, 11:1. http://www. reproductive-health-journal. com/content/11/1/1. Accessed July 13, 2017.

18 Charyeva, Z., O. Oguntunde, N. Orobaton, E. Otolorin, F. Inuwa, O. Alalade, D. Abegunde, et S. Danladi. 2015. "Task shifting provision of contraceptive implants to community health extension workers: Results of operations research in northern Nigeria," Global Health Science and Practice, 3(3): 382-394.

19 High-Impact Practices in Family Planning (HIPs). 2015. "Community health workers: Bringing family planning services to where people live and work." Washington, DC: USAID. http://www. fphighimpactpractices.org/resources/ community-health-workersbringing-family-planning-services-where-people-live-and-work.

20 Hoke, T., A. Brunie, K. Krueger, C. Dreisbach, A. Akol, N.L. Rabenja, A. Olawo, et J. Stanback. 2012. "Community-based distribution of injectable contraceptives," International Perspectives on Sexual and Reproductive Health 38(4): 214-219.

21 High-Impact Practices in Family Planning (HIP). 2013. "Drug shops and pharmacies: Sources for family planning commodities and information. "Washington, DC: USAID. http://www. fphighimpactpractices.org/resources/drug-shops-and-pharmaciessources-family-planning-commodities-and-information.

22 High-Impact Practices in Family Planning (HIPs). 2012. "Investing in contraceptive security and strengthening health systems." Washington, DC: USAID.

23 Costello, M., M. Lacuesta, S. RamaRao, et A. Jain. 2001. "A clientcentered approach to family planning: The Davao Project," Studies in Family Planning, 32(4): 302-314.

24 Sathar, Z., A. Jain, S. RamaRao, M. ul Haque, et Jacqueline Kim. 2005. "Introducing client-centered reproductive health services in a Pakistani setting," Studies in Family Planning, 36(3): 221-234.

25 Jain, A. 2016. "Examining progress and equity in information received by women using a modern method in 25 developing countries," International Perspectives on Sexual and Reproductive Health, 42(3): 131-140.

26 Shattuck, D., B. Perry, C. Packer, et D. Chin Quee. 2016 "A review of 10 years of vasectomy programming and research in low-resource settings," Global Health: Science and Practice, 4(4): 647-660. http://doi.org/10.9745/GHSP-D-16-00235.
27 Shelton, J.D., et R. Jacobstein. 2016. "Vasectomy: A long, slow hau to successful takeoff," Global Health: Science and Practice, 4 (4): 514-517. http:// dx.doi.org/10.9745/GHSP-D-16-00355.

28 Belaid, L. A. Dumont, N. Chaillet, A. Zertal, V. De Brouwere, et V. Ridde. 2016. "Implementation and effects of demand generation interventions in low- and middle-income family planning programs: A systematic review," Cahiers REALISME, No. 6, avril. http://www. equitesante. org/cahiers- realisme-numero-6-implementation-andeffects-of- demand-generation-interventions-in-low-and-middleincome-family- planning-programs-a-systematic-review/.

Auteurs : Heather Clark, Saumya Ramarao, Catherine Unthank, Population Council ; Kazuyo Machiyama, London School of Hygiene and Tropical Medicine ; Nandita Thatte, Organisation mondiale de la Santé

Ce document fait partie de l'un des sept Résumés de preuves sur la planification familiale préparés dans le cadre du Sommet de la planification familiale tenu à Londres le 11 juillet 2017. Les résumés mettent en exergue les éléments de preuve et fournissent des considérations sur la recherche et les programmes pour améliorer l'accès à la planification familiale et réduire les grossesses non désirées. Les considérations relatives aux programmes sont basées sur les opinions expertes des auteurs, qui ont procédé à des examens documentaires sur la base des preuves existantes.

\section{Résumés de preuves sur la planification familiale}

- Accélérer l'adoption de la planification familiale volontaire et fondée sur les droits dans les pays en développement (vue d'ensemble) (mise à jour octobre 2018)

- Financement de la planification familiale (mise à jour octobre 2018)

- Réduction des grossesses précoces et involontaires chez les adolescentes (mise à jour octobre 2018)

- Amélioration de la prestation de services de planification familiale pendant les crises humanitaires

- Assurer la sécurité des contraceptifs par des chaînes d'approvisionnement efficaces

- Élargissement du choix des contraceptifs (mise à jour octobre 2018)

- Partenariat avec le secteur privé pour renforcer la fourniture de la contraception

Seuls les auteurs sont responsables des opinions exprimées dans cet article qui ne représentent pas nécessairement les opinions, décisions ou politiques des institutions avec lesquelles ils sont affiliés.

La présente traduction n'a pas été établie par l'Organisation mondiale de la Santé (OMS). L'OMS ne saurait être tenue pour responsable du contenu ou de l'exactitude de la présente traduction. L'édition originale anglaise est l'édition authentique qui fait foi.

Family Planning Evidence Brief-Accelerating uptake of family planning: WHO/RHR/17.07

Family Planning Evidence Brief-Partnering with the private sector: WHO/RHR/17.08

Family Planning Evidence Brief-Ensuring contraceptive security through effective supply chains: WHO/RHR/17.09

Family Planning Evidence Brief-Reducing early and unintended pregnancies among adolescents: WHO/RHR/17.10

Family Planning Evidence Brief-Reducing early and unintended pregnancies among adolescents: programme priorities: WHO/ RHR/17.11

Family Planning Evidence Brief-Ensuring adequate financing of family planning commodities and services: WHO/RHR/17.12

Family Planning Evidence Brief-Improving family planning service delivery in humanitarian crises: WHO/RHR/17.13

Family Planning Evidence Brief-Expanding contraceptive choice: WHO/RHR/17.14

Ce document a été financé par UK AID du gouvernement britannique toutefois, les opinions exprimées ne reflètent pas nécessairement les politiques du gouvernement britannique.

Préparé en juillet 2017. Mis à jour octobre 2018 